\title{
Extra-anatomic Left Carotico-Subclavian Artery Bypass Technique in a patient with Subclavian Steal Syndrome: Case report
}

\author{
Ertan Demirdaş ${ }^{1}$, Kıvanç Atılgan², Zafer Cengiz Er ${ }^{3}$, Ferit Çiçekçioğlu ${ }^{1}$
}

\footnotetext{
1) Bozok University Medicine Faculty Cardiovascular Surgery Department, Prof.Dr., Yozgat-Turkey

2) Bozok University Medicine Faculty Cardiovascular Surgery Department, Assist.Dr., Yozgat-Turkey

${ }^{3)}$ Bozok University Medicine Faculty Cardiovascular Surgery Department, Op. Dr., Yozgat-Turkey
}

\section{Abstract}

Subclavian steal syndrome (SSS) is characterized by total occlusion of proximal part of subclavian artery resulting with retrograde flow of vertebral artery. The main risk factors in etiology are hypercholesterolemia and atherosclerosis. Congenital left subclavian artery stenosis and patent ductus arteriosus accompanying aortic coarctation, posttraumatic injuries, thromboembolism and arteritis are some of the rare factors causing SSS. SSS is usually an asymptomatic disease. However, the major symptoms are dizziness, claudication intermittent of upper extremity, headache and paresthesia in distal part of upper extremities. We are presenting a 57-year-old man presenting with a claudication intermittent on the left arm, paresthaesia in fingers, dizziness and headache for a three year time. After applying a computerized tomographic angiography, we observed that the left subclavian artery was totally occluded at the origin point. There are several surgical approaches for the treatment of this syndrome. In this case, we preferred the carotico-subclavian bypass technique with supraclavicular approach due to its low rates of mortality and morbidity, and high rates of long term durability.

Key words: Graft, carotid artery, subclavian steal, supraclavicular 


\section{Introduction}

Subclavian steal syndrome (SSS) is usually an asymptomatic disease characterized by total occlusion etiology of proximal part of subclavian artery. ${ }^{(1)}$ SSS was first described in the early 20th century and many of researches have been made both in diagnosing and treating areas. It is well known that SSS is a consequence of retrograde flow of vertebral artery following the total occlusion of proximal part of subclavian artery. ${ }^{(2)}$ The main risk factors in etiology are hypercholesterolemia and atherosclerosis. ${ }^{(2-4)}$ It is also possible to observe SSS due to congenital left subclavian artery stenosis and patent ductus arteriosus accompanying aortic coarctation. ${ }^{(5,6)}$ Besides, posttraumatic injuries, thromboembolism and arteritis are some of the rare factors causing SSS. ${ }^{(1-3)}$

SSS is usually an asymptomatic disease. However, the major symptoms are dizziness, claudication intermittent of upper extremity, headache and paresthesia in distal part of upper extremities. SSS is often diagnosed incidentally by means of observing the retrograde flow of vertebral artery in colored doppler ultrasonography of carotid and vertebrobasillary arteries getting applied to the patients having dizziness and observing a gradient in tension arterial (TA) pressures between two upper extremities.

Image 1. Preoperative Computerized Tomographic Angiography image

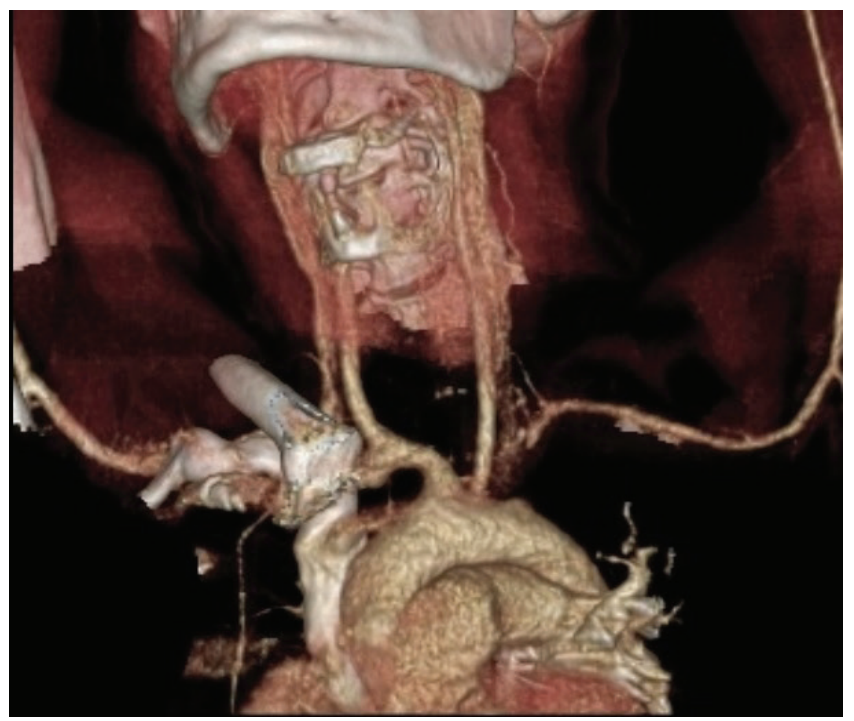

\section{Case Report}

A 57-year-old male with a history of claudication intermittent on left arm, paresthesia in dita, disseness and headache for three years applied to our department. Distal pulses were not palpable on the left upper extremity and TA was $130 / 80 \mathrm{mmHg}$ on right arm and $60 / 30 \mathrm{mmHg}$ on left arm. On the computerized tomographic angiography (CTA), we observed that the first $3 \mathrm{~cm}$ part of the subclavian artery begining from the aortic arcus origin was totally occluded.(Image 1)

\section{Operation Technique}

Invasive arterial monitorization was applied from right upper extremity. The patient was positioned as the head in hyperextension and the face looking to the opposite side, left shoulder was elevated and the upper extremities were pulled down. An $8 \mathrm{~cm}$ left supraclavicular incision was applied, common carotid and subclavian arteries were dissected gently and the proximal and distal parts were fixed with tapes. After systemic heparinization common carotid artery was clamped. Following arteriotomy the proximal segment of an 8 $\mathrm{mm}$ PTFE graft was anastomosed to the artery. After declamping, subclavian artery was clamped and arteriotomy was applied. Distal segment of PTFE graft was anastomosed to the subclavian artery.(Image 2) After

\section{Image 2. Intraoperative image of the graft}

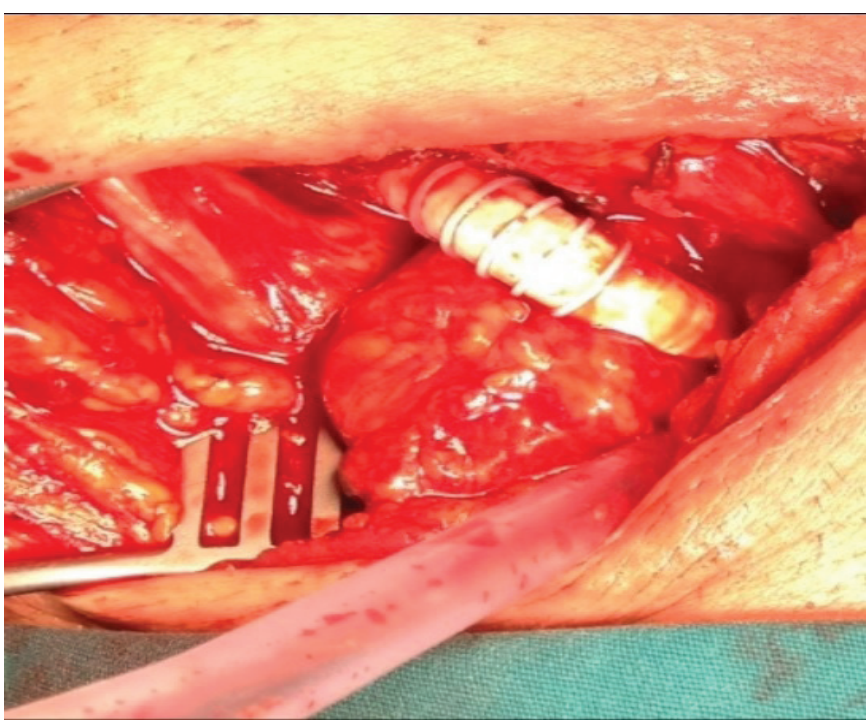


the operation on both sides of upper extremities distal pulses were palpable. The patient was discharged on the postoperative 6th day without any complication.

\section{Discussion}

Subclavian steal syndrome (SSS) is characterized by total occlusion of proximal part of subclavian artery resulting with retrograde flow of vertebral artery. The retrograde vertebral artery flow may cause variable symptoms like cerebellar ischaemia (dizziness, blumia), cladication intermittent and paresthaesia on the upper extremity.

There are different types of surgical techniques, almost all of which get a remarkable success in eliminating the symptoms, and surgery should be a treatment approach only in symptomatic patients. The first successful transtorasic intervention for a subclavian artery obstruction was achieved by De Bakey et al in 1958 . (7) In 1964 Parrot described the carotico-subclavian artery bypass procedure. ${ }^{(8)}$ Carotico-subclavian bypass, subclavio-subclavian bypass, axillo-subclavian bypass and subclavio-carotid transposition techniques are described as extra-anatomic procedures, and aortasubclavian bypass and endarterectomy are descired as anatomic procedures. Transthoracic incision techniques are not preferred anymore due to high mortality and morbidity rates. ${ }^{(9)}$

Percutaneous transluminal angioplasty and stenting
(PTAS) are some of the treatment approaches as an alternative to surgery. Especially PTAS have become the first option in SSS cases occuring as a result of vasculitis or radiotherapy. ${ }^{(10)}$ However, the long term durability of surgery is superior to PTAS. ${ }^{(11)}$

Carotico-subclavian artery bypass technique is an extra-anatomic bypass process and has a low morbidity and mortality rates. Vogt et al, reported the mortality rate of intrathoracic techniques as $15 \%$ and of extraanatomic techniques as $0 \%$, in 1982. ${ }^{(12)}$ Saphenous and artificial grafts can be used for bypassing. However, 5 year durability rates are reported as $95,2 \%$ for PTFE grafts, $83,9 \%$ for Dacron grafts and $64 \%$ for saphenous vein grafts. ${ }^{(13)}$ In our case we preferred an $8 \mathrm{~mm}$ PTFE graft.

In recent researches, carotico-subclavian artery bypass technique is described as a process having low mortality and morbidity, and long term survival rates. $(14,15)$ Normal carotid artery anatomy is a necessity for this technique, otherwise it is possible to cause a carotid artery steal syndrome. ${ }^{(16-18)}$

\section{Conclusion}

Extra-anatomic carotico-subclavian artery bypass technique is a relatively easy process in comparison to other techniques, and has better mortality and morbidity rates, because of that we prefer and recommend this technique as a first option for patients having SSS. 


\section{References}

1. Potter BJ, Pinto DS. Subclavian steal syndrome. Circulation, 2014 jun $3 ; 129(22): 2320-3$

2. Abova V,Kamineni A, Allison Ma,Mc Dermott MM,Crouse JR, Ni h,Szklo et al. The epidemiology of subclavian stenosis and its association with markers of subclinical atherosclerosis: The Multi-Etnic study of atherosclerosis (MESA). Atherosclerosis. 2010;211:266-70.

3. Labropoulos N, Nandivada P, Bekelis K. Prevalence and impact of the subclavian steal syndrome. Ann Surg. 2010;252:166-70.

4. Harper C, Cardullo PA, Weyman AK, Patterson RB Transcranial Doppler ultrasonography of the basilar artery in patients with retrograde vertebral artery flow. J Vasc Surg. 2008;48:859-64.

5. Gerber N. Congenital atresia of the subclavian artery producing an subclavian steal syndrome. Amer j dis child 1967;113:709-13.

6. Daves j,Treger A. Vertebral grand larcency. Circulation 1964;29:91-3.

7. DeBakey ME, Morris GC, Jordan GL, Cooley DA. Segmental tromboobliterative disease of branches of aortic arch. JAMA 1958;166:9881003 .

8. Parrot JD. The subclavian steal sendrome. Arch Surg 1964;88:661-5.

9. Fields WS, Lemak HA. Joint study of extracranial Arterial Occlusion, VII.Subclavian steal - a review of 168 cases. JAMA 1972;222:1139-43.

10. Kim HJ, Lee CS, Kim JS, Know SU, Kim JL, Park JW, et al. Outcomes after endovascular treatment of symptomatic patients with Takayasu's arteritis. Interv Neuroradiol 2011;17:252-60.

11. Tetik Ö, YAŞA H, Demir T, Gürbüz A. Subklavyan Steal sendromu: 3 olgu nedeniyle. İzmir Atatürk Eğitim ve araştırma Hastanesi Tıp dergisi 2004;139-41

12. Vogt DP, Hertzer NR, O’Hara PJ, Beven EG. Brachiocephalic arterial reconstruction. Ann surg. 1982;196:541-52.

13. Law MM, Colburn MD, Moore WS, Quinones-Baldrich WJ, Machleder HI, Gelabert HA. Carotid-subclavian bypass for brachiocephalic occlusive disease. Stroke 1995;26:1565-71.

14. Aburahma AF, Robinson PA, Jennings TG. Carotidsubclavian bypass grafting with polytetrafluoroethylene grafts for syptomatic subclavian artery stenosis or occlusion: A 20-year experience. J vasc surg 2000;32:411-9.

15. Vitti MJ, Thompson BW, Read RC, et al. Carotidsubclavian bypass: A twenty-two year experience. J Vasc surg 1994;20:411-8.

16. Lord RS, Ehrenfeld WK. Carotid-subclavian bypass: A hemodynamic study. Surgery 1969;66:521-6.

17. Cook CH, Stemmer EA, Connolly JE. Effect of peripheral resistance on carotid blood flow after Carotid-subclavian bypass. Arch surg 1972;105:9-13.

18. Swayngim DM Jr, Avellone JC. Hemodynamic consequences of axilloaxillary bypass J cardiovasc surg 1982;23:65-8.
Received: 09/02/2017

Accepted: 31/08/2017

Published: 15/09/2017

Disclosure and conflicts of interest:

The authors declare no conflict of interest.

Corresponding author:

Prof. Dr. Ertan Demirdaş

Mail: dr.ertandemirdas@gmail.com 